\title{
Survival Outcomes of Advanced and Recurrent Cervical Cancer Patients Treated with Chemotherapy: Experience of Northern Tertiary Care Hospital in Thailand
}

\author{
Kuanoon Boupaijit, Prapaporn Suprasert*
}

\begin{abstract}
Chemotherapy is the primary treatment for advanced and recurrent cervical cancer. To evaluate the survival outcomes of chemotherapy and the prognostic factors in this setting, we conducted a retrospective study by reviewing the medical records of advanced and recurrent cervical cancer patients treated with systemic chemotherapy at our institute between January, 2008 and December, 2014. One hundred and seventy-three patients met the criteria with a mean age of 50.9 years. $4.1 \%$ of them were HIV positive. The most common initial stage was stage IVB $(30.1 \%)$ and the most common histology was squamous cell carcinoma (68.6\%). Ninety-two $(53.2 \%)$ patients were previously treated with concurrent chemoradiation with $53 \%$ developing combined sites of recurrence. The median recurrence free interval was 16.7 months. Cisplatin +5 fluorouracil (5FU) $(53.2 \%)$ was the most frequent first line chemotherapy followed by carboplatin + paclitaxel (20.2\%) with an objective response of $39.3 \%$. Seventy-two patients received subsequent chemotherapy. The median overall survival of all studied patients was 13.2 months. Only a recurrence free interval of less than 12 months was an independent prognostic factor for survival outcome. In conclusion, chemotherapy treatment for advanced and recurrent cervical cancer patients showed modest efficacy with a shorter recurrence free survival less than 12 months as a significant poor prognosis factor.
\end{abstract}

Keywords: Cervical cancer - advanced stage - recurrence - chemotherapy - prognosis

Asian Pac J Cancer Prev, 17 (3), 1123-1127

\section{Introduction}

Cervical cancer is the third most common female cancer in the world (Ferlay et al., 2012). Primary treatment consists of surgery, concurrent chemoradiation and systemic chemotherapy for early, locally advanced and advanced stage, respectively with impressive outcomes. However, the recurrence rate and persistent disease still developed in about one-third of all cervical cancer patients especially in the advanced stage (Peiretti et al., 2012; Micha et al., 2015). The main treatment for these recurrent, persistent and also the advanced stage patients is cisplatin-based chemotherapy ((Kamura and Ushijima ,2013; Mackay et al., 2015). Various drugs were tested to combine with cisplatin such as ifosfamide, 5-fluorouracil (5FU), paclitaxel, gemcitabine, vinorelbine, topotecan with the aim to improve survival in the clinical trials and only paclitaxel seemed to gain a survival benefit and showed the least adverse effects than other drugs with the response rate of $29 \%$ and the median progression-free survival and overall survival were 5.8 months and 12.9 months, respectively (Monk et al., 2009). Thus, cisplatin combined with paclitaxel was accepted as the standard treatment for these recurrence patients (Pfaendler and Tewari, 2016). After that, a Japanese study showed the non-inferior outcomes of carboplatin plus paclitaxel when compared to cisplatin plus paclitaxel with less adverse effects especially in patients who previously received cisplatin (Kitagawa et al., 2015) The combination of carboplatin plus paclitaxel has been used more often. In Thailand, most recurrent cervical cancer patients received various regimens depending on the performance status and physician preference. However, the outcomes of these patients were still limited. We conducted this retrospective study with the primary outcome to identify survival data and the secondary outcomes and to find the independent poor prognostic factors.

\section{Materials and Methods}

After the protocol was approved by the local ethics committee, the medical records of the recurrent, the persistent and the advanced cervical cancer patients who were treated with chemotherapy at Chiang Mai University 
Hospital between January, 2008 and December, 2014 were retrospectively reviewed. The patients who were previously treated with chemotherapy except in the setting of neoadjuvant or concurrent chemoradiation were excluded. The patients were diagnosed as recurrent, persistent or advanced by physical examination combined with the appropriated imaging. The clinical data, the histology, the staging, the initial treatment methods, the recurrence site, the progression-free interval defined as the time from the completed initial treatment to the time of recurrence and the details of chemotherapy regimens were collected.

The first line chemotherapy was given every three to four weeks until progression or unacceptable toxicity. The further chemotherapeutic regimens were subsequently given due to progression or non-response to first line treatment. The WHO criteria were used to evaluate the response of chemotherapy. After completion of the course of chemotherapy regimen, the patients were followed up with gynecologic oncologists for physical and pelvic examinations every three months in the first year, every four months in the second year and every six months in the third to fifth year, then annually.

Statistical analysis of the data was carried out using IBM SPSS statistic for Window program (Version 22). Descriptive data of all studied patients were presented as means with range and discrete data were reported as number and percentages. The overall survival defined as the time between the month of initial chemotherapeutic treatment and the month of patients' death or last contact was estimated by the Kaplan-Meier method. Factors influencing survival were analyzed using Cox's proportional hazard regression analysis. Statistical significance was noted when a P-value was less than 0.05 .

\section{Results}

There were 173 patients who met the inclusion criteria in the studied period. The details of the clinical data were noted in Table 1 . The mean age of the initial diagnosis was 48.4 years and the mean age of the development of

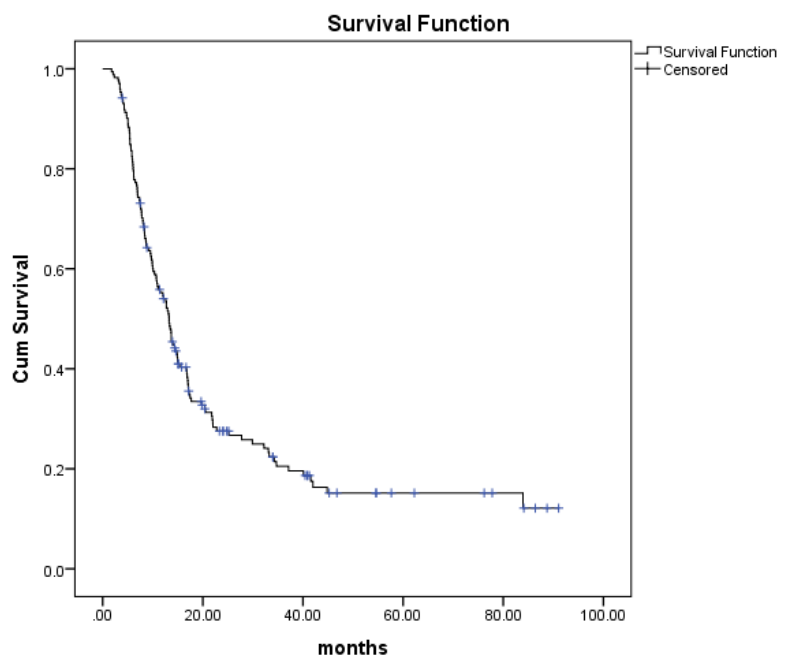

Figure 1. Overall Survival. 5 Year Overall Survivals = $15.2 \%$, Median Overall Survival = 13.21 Months recurrence was 50.9 years. Of those patients, $4.1 \%$ were HIV positive. The most common initial stage was stage IVB, 30\% who all received chemotherapy as their primary treatment. Thirty-seven percent of the studied patients received concurrent-chemo-radiation as primary treatment and $16.2 \%$ received chemo- radiation after surgery. The most common histology was squamous cell carcinoma that was in found nearly $70 \%$ of the patients.

Regarding recurrence site, half of the patients developed combined local and distant metastatic sites.

Table 1. Patients Clinical Characteristics $(N=173)$

\begin{tabular}{|c|c|}
\hline & $\mathrm{N}(\%)$ \\
\hline $\begin{array}{l}\text { Mean Age of First Diagnosis (+2 SD): } \\
\text { Years }\end{array}$ & $48.44(+9.42)$ \\
\hline $\begin{array}{l}\text { Mean Age of Start Treatment After } \\
\text { Recurrence (+ } 2 \text { SD): Years }\end{array}$ & $50.90(9.20)$ \\
\hline Positive HIV & $7(4.1)$ \\
\hline Underlying Disease & $47(27.17)$ \\
\hline \multicolumn{2}{|l|}{ Initial Stage } \\
\hline IA & $2(1.2)$ \\
\hline IB1 & $27(15.6)$ \\
\hline IB2 & $7(4.0)$ \\
\hline IIA1 & $4(2.3)$ \\
\hline IIA2 & $4(2.3)$ \\
\hline IIB & $39(22.5)$ \\
\hline IIIA & $3(1.7)$ \\
\hline IIIB & $33(19.1)$ \\
\hline IVA & $2(1.2)$ \\
\hline IVB & $52(30.1)$ \\
\hline \multicolumn{2}{|l|}{ Histology } \\
\hline Squamous Cell Carcinoma & $119(68.8)$ \\
\hline Adenocarcinoma & $30(17.3)$ \\
\hline Clear Cell Carcinoma & $2(1.2)$ \\
\hline Small Cell Neuroendocrine & $12(6.9)$ \\
\hline Other & $10(5.8)$ \\
\hline \multicolumn{2}{|l|}{ Initial Treatment } \\
\hline None & $45(26.0)$ \\
\hline Concurrent Chemo radiation & $64(37.0)$ \\
\hline $\begin{array}{l}\text { Surgery Followed by Concurrent Chemo } \\
\text { radiation }\end{array}$ & $28(16.2)$ \\
\hline Surgery & $15(8.7)$ \\
\hline Palliative Radiation & $9(5.2)$ \\
\hline Radiation & $6(3.5)$ \\
\hline $\begin{array}{l}\text { Neoadjuvant Chemotherapy Followed by } \\
\text { Radiation }\end{array}$ & $5(2.9)$ \\
\hline $\begin{array}{l}\text { Neoadjuvant Chemotherapy Followed by } \\
\text { Surgery }\end{array}$ & $1(0.6)$ \\
\hline \multicolumn{2}{|l|}{ Recurrent Site } \\
\hline Local & $40(23.1)$ \\
\hline Regional & $6(3.5)$ \\
\hline Distant & $34(19.7)$ \\
\hline Combined & $93(53.8)$ \\
\hline \multicolumn{2}{|l|}{ Prior Treatment After Recurrence } \\
\hline None & 161(93.1) \\
\hline Surgery & 2(1.2) \\
\hline Radiation & $10(5.8)$ \\
\hline
\end{tabular}


Table 2. Chemotherapy and Outcome

\begin{tabular}{lcccc}
\hline \multicolumn{1}{c}{ Line of Treatment } & Drugs & $\mathrm{N}(\%)$ & Response & $\mathrm{N}(\%)$ \\
\hline First Line $(\mathrm{N}=173)$ & Cisplatin + 5FU & $92(53.2)$ & Progression & $37(21.4)$ \\
Median Cycle (Range) & Carboplatin + Paclitaxel & $35(20.2)$ & Stable of Disease & $16(9.2)$ \\
0 & Cisplatin + Paclitaxel & $5(2.9)$ & Partial Response & $47(27.2)$ \\
& Cisplatin + Etoposide & $3(1.7)$ & Complete Response & $21(12.1)$ \\
& Carboplatin & $18(10.4)$ & Unknown (Loss to FU) & $4(2.3)$ \\
& Cisplatin & $13(7.5)$ & & \\
& Ifosfamide & $1(0.6)$ & & $37(51.4)$ \\
& Other & $6(3.5)$ & & $16(22.2)$ \\
Second Line $(\mathrm{N}=72)$ & Cisplatin + 5FU & $12(16.7)$ & Progression & $11(15.3)$ \\
Median Cycle (Range) $=3(1-8)$ & Carboplatin + Paclitaxel & $23(31.9)$ & Stable of Disease \\
& Cisplatin + Paclitaxel & $2(2.8)$ & Partial Response & $4(5.6)$ \\
& Cisplatin + Etoposide & $2(2.8)$ & Complete Response \\
& Carboplatin & $7(9.7)$ & Unknown (Loss to FU) & $4(5.6)$ \\
& Cisplatin & $6(8.3)$ & & \\
Forth Line $(\mathrm{N}=2)$ & Ifosfamide & $15(20.8)$ & & \\
Median Cycle (Range) 1.5(1-2) & Other & $5(6.9)$ & & \\
Median Cycle (Range) $=3(1-6)$ & Cisplatin + 5FU & $1(6.3)$ & Progression & $7(43.8)$ \\
& Carboplatin + Paclitaxel & $4(25.0)$ & Stable Of Disease & $5(31.3)$ \\
& Ifosfamide & $8(50.0)$ & Partial Response & $2(12.5)$ \\
& Other & $3(18.8)$ & Complete Response & $1(6.3)$ \\
& Ifosfamide & & Unknown (Loss To FU) & $1(6.3)$ \\
& 5FU & $1(0.6)$ & Progression & $1(50.0)$ \\
& & $1(0.6)$ & Stable Of Disease & $1(50.0)$ \\
\hline
\end{tabular}

Table 3. Cox Proportional Hazard Analysis for Prognostic Factors Affecting Survival

\begin{tabular}{|c|c|c|c|}
\hline Factor & $\mathrm{N}(\%)$ & Median OS (Months) & P Value \\
\hline \multicolumn{3}{|l|}{ Received PT Regimen } & 0.259 \\
\hline Yes & $40(23.1)$ & 12.94 & \\
\hline No & $133(76.9)$ & 13.27 & \\
\hline \multicolumn{3}{|l|}{ Age } & 0.285 \\
\hline Less Than or Equal 60 Years & $155(89.6)$ & 13.21 & \\
\hline More Than 60 Years & $18(10.4)$ & 11.36 & \\
\hline \multicolumn{3}{|l|}{ Histology } & 0.235 \\
\hline SCCA & $119(68.6)$ & 13.27 & \\
\hline Non-SCCA & $54(31.2)$ & 12.71 & \\
\hline \multicolumn{3}{|l|}{ Previous Received Chemotherapy } & 0.59 \\
\hline Never & $88(50.9)$ & 13.27 & \\
\hline Yes & $85(49.1)$ & 12.94 & \\
\hline \multicolumn{3}{|l|}{ Treatment After Metastasis } & 0.62 \\
\hline None & $161(93,2)$ & 12.94 & \\
\hline Yes & $12(6.9)$ & 17.25 & \\
\hline \multicolumn{3}{|l|}{ Site of Recurrence } & 0.305 \\
\hline Local and Regional & $46(26.6)$ & 12.94 & \\
\hline Distant and Combined & $127(73.4)$ & 13.27 & \\
\hline \multicolumn{3}{|c|}{ Progression Free Interval (Only Recurrence Patients; N = 125) } & 0.03 \\
\hline Less Than or Equal 12 Months & $48(27.7)$ & 8.77 & \\
\hline More Than 12 Months & $77(44.5)$ & 13.47 & \\
\hline \multicolumn{3}{|l|}{ Prior Radiation Treatment } & 0.231 \\
\hline Yes & $112(64.7)$ & 12.12 & \\
\hline No & $61(35.3)$ & 15.38 & \\
\hline
\end{tabular}

PT = carboplatin + paclitaxel; SCCA = squamous cell carcinoma

Only $23 \%$ revealed local recurrence and $6.8 \%$ of these recurrence patients received subsequent surgery (two cases) and radiation (ten cases) before the chemotherapy was given. The median recurrence free interval after completing their final treatment before they received chemotherapy was 16.7 months with a range of 1-337.2 
months.

The details of chemotherapy and the outcomes were listed in Table 2. The median cycles of the first line, second line, third line and fourth line chemotherapy were 5, 3,3, and 1.5 cycles, respectively. Almost $50 \%$ of the studied patients received cisplatin plus 5 fluorouracil and about $20 \%$ received carboplatin and paclitaxel as first line chemotherapy. The complete response and partial responses in patients who received cisplatin $+5 \mathrm{FU}$ were $15.2 \%$ and $27.2 \%$ while the complete response and partial response in patients who received carboplatin plus paclitaxel were $5.7 \%$ and $28.6 \%$, respectively. However, the complete response of all first line chemotherapy was $12.1 \%$ and the partial response was $27.2 \%$. The median overall survival of patients received non paclitaxel regimen and carboplatin plus paclitaxel were 13.2 months and 12.94 months, respectively. Seventy-two patients were given second line chemotherapy and the most frequent regimen was carboplatin plus paclitaxel. The complete response was $5.6 \%$ and the partial response was $15.3 \%$. Sixteen patients received third line chemotherapy with the most frequent regimen ifosfamide with the complete response $6.3 \%$ and partial response was $12.5 \%$. Only two patients received fourth line chemotherapy with ifosfamide and $5 \mathrm{FU}$ in each patient. One of them achieved stable of their disease while the other developed progression. Finally, 133 patients $(76.9 \%)$ died of their disease with the median overall survival of 13.2 months and five-year overall survival rate was $15.2 \%$ as showed in Figure 1.

Regarding prognostic factors, the significant independent prognostic factor for survival outcome was only the recurrence free interval less than 12 months after complete initial treatment that had median overall survival only 8.77 months compared with 13.47 months in those patients with progression free interval longer than 12 months. The other prognostic factors including: elderly patients, treatment with platinum and paclitaxel, non-squamous cell histology, previous treatment with chemotherapy, former treatment after metastasis detection before receiving chemotherapy, and the recurrence site were not significant in Cox regression analysis as noted in Table 2.

\section{Discussion}

The five-year overall survival of the advanced and recurrent cervical cancer who were treated with chemotherapy in the present study was $15.2 \%$ and the median overall survival was 13.2 months that corresponded with the previous literature review that showed the five year- survival rate between 5\% and $15 \%$ and the median overall survival at 12 months (Mackay et al., 2015). The most common first line chemotherapy regimen in the present study was cisplatin plus $5 \mathrm{FU}$ that achieved objective response at $42.4 \%$. This response rate was closely to Kaern et al. that revealed the objective response of cisplatin plus $5 \mathrm{FU}$ at $48 \%$ in 72 recurrent cervical cancer patients (Kaern et al., 1996). However, various drugs were tested in combination with cisplatin compared to cisplatin alone such as ifosfamide, paclitaxel, gemcitabine, vinorelbine, topotecan in Phase III setting for the purpose of improving survival rates (Kamura $\mathrm{T}$ and Ushijima K, 2013, Eskander and Tewari 2014) and finally only cisplatin combined with topotecan showed significantly better results than cisplatin alone in term of response rate, progression free and overall survival (Long et al., 2005) while cisplatin plus paclitaxel showed to be significantly better only in response rate and progression free survival but not for overall survival (Moore et al., 2004). After that, the Gynecologic Oncology Group (GOG) conducted a four-arm Phase III study comparing cisplatin plus paclitaxel with cisplatin plus topotecan, cisplatin plus gemcitabine, and cisplatin plus vinorelbine and was found to be non-inferior in each arm but the trend of response rate, progression-free survival and overall survival favored cisplatin plus paclitaxel. Therefore, this combination regimen became the standard therapy in this setting in the United States (Eskander and Tewari, 2014). Afterwards, to reduce toxicity of cisplatin, carboplatin, the cisplatin derivative that showed minimal renal toxicity was given combined with paclitaxel and found non-inferior outcomes when compared with cisplatin plus paclitaxel especially in patients who were previously treated with cisplatin in the Japan Clinical Oncology Group trial (Kitagawa et al., 2015). To date, carboplatin plus paclitaxel regimen was in popular use in this setting. In the present study, about $20 \%$ of the studied patients received platinum plus paclitaxel that mostly was carboplatin plus paclitaxel and showed median overall at 12.94 months that was close to the GOG 204 trial (Monk et al., 2009) that showed the median overall survival at 12.84 months but shorter than the Japanese report that showed the median overall survival at 18.3 months (Kitagawa et al., 2015). The difference might be from variation in the number and character of the recruited patients. Recently, Bevacizumab, a humanized anti-vascular endothelial growth factor monoclonal antibody showed significant survival benefit with the improvement of 3.7 months in the median overall survival when given with cisplatin plus paclitaxel regimen or paclitaxel plus topotecan regimen in the GOG 240 randomized trial (Tewari et al., 2014). Unfortunately, we did not have any experience with bevacizumab in this present study.

Regarding the poor prognostic factors for survival outcome, the present study found that only the interval time between initial treatment and the developed of recurrence or progression of disease less than 12 months was the independent poor prognostic factor while receiving non-paclitaxel regimen, the age younger than 60 years, the non-squamous cell histology, the previous treatment with chemotherapy, the received prior treatment after recurrence detection and the location of recurrence were not significantly factors. Moore et al. (Moore et al., 2010 ) reported five independent prognostic factors for survival outcomes of cisplatin-based chemotherapy in 428 advanced cervical cancer patients from three GOG protocols (GOG 110,169 and 179). These factors included African-American, performance status more than zero, pelvic disease, prior radiotherapy, and time interval from diagnosis to first recurrence less than 12 months. Thus, the interval time to developed recurrence less than 12 months was a similar independent prognostic factor while 
prior cisplatin, pelvic disease, prior radiotherapy did not significant affect survival outcomes in this report. These inconsistent factors might be due to the low number of subjects studied that might not have enough power to show differences.

Concerning treatment following progression from first line chemotherapy, the present study showed a very poor response in the later chemotherapy regimen. The decreased effectiveness of the subsequent various chemotherapy regimens was due to drug resistance. A molecular targeting agent might be one solution to improve this treatment outcome in the future (Kamura and Ushijima ,2013).

The strength of the present study was that it was conducted in a single institution that could decrease the variation between the initial and subsequent treatments. Therefore, our results would be beneficial for developing a national policy regarding a standard chemotherapy regimen for this disease. However, one limitation of the present study was the limited number of studied patients that might affect the analysis of the prognostic factors.

In conclusion, the survival outcome of chemotherapy in patients with advanced, persistent and recurrence cervical cancer patients was not impressive with the median overall survival only 13 months. The poor independent prognostic factor for survival outcome was a shorter than one year of recurrence-free interval time.

\section{Acknowledgements}

We wish to thank the National Research University Project under Thailand's Office of Higher Education Commission and Chiang Mai University for their financial support of this project.

\section{References}

Ferlay J, Soerjomataram I, Ervik M, et al (2013). GLOBOCAN 2012 v1.0, Cancer Incidence and Mortality Worldwide: IARC CancerBase No. 11 [Internet]. Lyon, France: International Agency for Research on Cancer.

Eskander RN, Tewari KS (2014). Chemotherapy in the treatment of metastatic, persistent, and recurrent cervical cancer. Curr Opin Obstet Gynecol, 26, 314-21

Kaern J, Tropé C, Sundfoer K, et al (1996). Cisplatin/5fluorouracil treatment of recurrent cervical carcinoma: a phase II study with long-term follow-up. Gynecol Oncol, 60, 387-92.

Kamura T, Ushijima K (2013). Chemotherapy for advanced or recurrent cervical cancer. Taiwan J Obstet Gynecol, 52, 161-4.

Kitagawa R, Katsumata N, Shibata T, et al (2015). Paclitaxel Plus Carboplatin Versus Paclitaxel Plus Cisplatin in Metastatic or Recurrent Cervical Cancer: The Open-Label Randomized Phase III Trial JCOG0505. J Clin Oncol, 33, 2129-35.

Long HJ 3rd, Bundy BN, Grendys EC Jr, et al (2005). Randomized phase III trial of cisplatin with or without topotecan in carcinoma of the uterine cervix: a Gynecologic Oncology Group Study. J Clin Oncol, 23, 4626-33.

Moore DH, Blessing JA, McQuellon RP, et al (2004). Phase III study of cisplatin with or without paclitaxel in stage IVB, recurrent, or persistent squamous cell carcinoma of the cervix: a gynecologic oncology group study. J Clin Oncol,
22, 3113-9.

Monk BJ, Sill MW, McMeekin DS, et al (2009). Phase III trial of four cisplatin-containing doublet combinations in stage IVB, recurrent, or persistent cervical carcinoma: a gynecologic oncology group study. J Clin Oncol, 27, 4649-55.

Moore DH, Tian C, Monk BJ, et al (2010). Prognostic factors for response to cisplatin-based chemotherapy in advanced cervical carcinoma: a Gynecologic Oncology Group Study. Gynecol Oncol, 116, 44-9.

Micha JP, Sassoon AF, Wong H, et al (2015). Prolonged remission of recurrent cervical carcinoma following paclitaxel and carboplatin chemotherapy with paclitaxel maintenance chemotherapy. Anticancer Drugs, 26, 793-6.

Mackay HJ, Wenzel L, Mileshkin L(2015). Nonsurgical management of cervical cancer: locally advanced, recurrent, and metastatic disease, survivorship, and beyond. Am Soc Clin Oncol Educ Book, 299-309.

Peiretti M, Zapardiel I, Zanagnolo V, et al (2012). Management of recurrent cervical cancer: a review of the literature. Surg Oncol, 21, 59-66.

Pfaendler KS, Tewari KS (2016). Changing paradigms in the systemic treatment of advanced cervical cancer. Am J Obstet Gynecol, 214, 22-30.

Tewari KS, Sill MW, Long HJ 3rd, et al (2014). Improved survival with bevacizumab in advanced cervical cancer. $N$ Engl J Med, 370, 734-43. 